dose oral colchicine and hydroxychloroquine can be used in the management of recurrent acute CPP crystal arthritis to prevent recurrent flares. Based on current evidence, methotrexate cannot be recommended for the management of CPPD. However, there are anecdotal reports of beneficial effects of methotrexate in the management of chronic CPP crystal inflammatory arthritis, and, this agent holds promise for a role in the management of chronic CPP crystal arthritis which requires investigation in future studies. Radiosynovectomy may be effective in joints with chronic synovitis and CPPD. Individuals with young-onset CPPD, or polyarticular CPPD should be screened for inherited disorders such as ANKH gene mutations, Gitelman's syndrome; and for metabolic conditions such as hyperparathyroidism, haemochromatosis, hypomagnesemia and hypophosphatasia. When present, the underlying metabolic abnormality should be treated appropriately. In joints with end-stage osteoarthritis, the presence of CPPD does not adversely affect the outcomes of total joint replacement. There are anecdotal case-reports of radiographic articular chondrocalcinosis due to hyperparathyroidism or hypomagnesemia resolving after parathyroidectomy, or magnesium supplementation, to date, it has not been possible to dissolve CPPD in vivo with targeted drug therapy. Several candidate pharmaceutical agents that have the potential of dissolving CPPD have been identified, but, their clinical trial is generally precluded by the risk of systemic toxicity.

Conclusion: Although the pathogenesis of CPPD and CPP crystal mediated inflammation are well understood, its' management remains symptomatic. Expedited drug development programs and high quality randomised controlled trials are needed to advance the management of people with this disorder.

Disclosure of Interests: Abhishek Abhishek Grant/research support from: AstraZeneca and Oxfordlmmunotech, Grant/research support from: AstraZeneca and Oxford Immunotech, Speakers bureau: Menarini pharmaceuticals, Speakers bureau: Menarini pharmaceuticals

DOI: 10.1136/annrheumdis-2019-eular.8410

WEDNESDAY, 12 JUNE 2019

14:15:00 - 15:45:00

\section{Comorbidities in psoriatic arthritis}

\section{SP0009 PSORIATIC ARTHRITIS}

lain McInnes. University of Glasgow, Institute of Infection, Immunity, and Inflammation, Glasgow, United Kingdom

Background: Psoriasis and psoriatic arthritis ar associated with increased cardiovascular and cardio metabolic risk.

Objectives: To describe the prevalence and magnitude CV risk in psoriasis and psoriatic arthritis and to comment on the clinical therapeutic implications of this risk in our practice.

Methods: Review of literature concerning CV risk and interpretation of the same. Results: MACE are elevated over time in people with psoriasis and PsA. The mechanisms driving this are currently unclear but likely include both the impact of traditional risk factors e.g. dyslipidaemia, hypertension, obesity and novel risk factors e.g. mediated via increased inflammatory burden. Increased vigilance is required to detect this risk and a low index of suspicion should be introduced in practice. Aggressive management of CV risk should improve outcomes.

Conclusion: Recognising and treating factors mediating CV risk in psoriasis and psoriatic arthritis should comprise part of our routine clinical practice.

Disclosure of Interests: lain McInnes Grant/research support from: AstraZeneca, Celgene, Compugen, Novartis, Roche, UCB Pharma, Consultant for: AbbVie, Celgene, Galvani, Lilly, Novartis, Pfizer, UCB Pharma

DOI: 10.1136/annrheumdis-2019-eular.8536

\section{SP0010 INFECTIOUS RISK AND MANAGEMENT OF VACCINATION}

Nico Wulffraat. Wilhelmina Children's hospital/UMC Utrecht, Pediatric rheumatology and Immunology, Utrecht, Netherlands

Background: O ver the years, awareness of vaccine-preventable infection in rheumatic diseases has increased. At the same time, the safety of vaccines is still a hot topic in the public arena. Large-scale registries with data on juvenile idiopathic arthritis or RA taking the combination of methotrexate and biologics have demonstrated an increased incidence of infections. EULAR recommendations for both vaccination in pediatric patients and adults with rheumatic diseases, provide evidence-based guidance based on data from published studies on safety and efficacy of vaccinations in adults and children with rheumatic conditions. Especially in children, few data exist on safety and efficacy of live-attenuated vaccines such as MMR. This is relevant given the decreasing vaccination coverage and occurrence of measles outbreakes

Objectives: To provide an overview of vaccines used in patients groups with rheumatic conditions under medication such as MTX and or biologicals. Also we seek for evidence on adverse events, protection rates and persistence of protective antibody titers over time. From these studies general guidelines are proposed.

Methods: Literature review of vaccination studies performed in adults and children. In addition the clinical practice of boostering of MMR in JIA patients under immunosuppressive therapy was investigated in several large academic pediatric centers across Europe. Thus 234 JIA patients were identified that received a MMR booster under MTX and or biologicals.

Results: EULAR recommendations are updated for children and adults. For most vaccines it is adivised to follow the national vaccination guidelines. Non-live vaccines can be safely provided to AIIRD patients under immunomodulating treatments, whereas the administration of live attenuated vaccines should be avoided under immunomodulating treatment, with the possible exceptions of herpes zoste and MMR. In 234 children with JIA who received the MMR booster while using MTX and or a Biological only 7 of adverse events were reported. Adverse events reported were mild (skin reaction, mild fever, urinary tract infection. There was no relation between disease activity, disease type or duration, sex, age and outcome of vaccinations.

Vaccination status of patients with rheumatic conditions should be assessed annually. Live attenuated vaccines should be avoided during the first 6 months of life in newborns of mothers treated with biologics during the second half of pregnancy.

Conclusion: The EULAR recommendations of 2011 are currently updated and will provide an up-to-date guidance on the management of vaccinations in patients with inflammatory rheumatic conditions. There is still a scartity of data on safety and efficay of live attenuated vaccines under biologicals. The next challenge is the implementation into the clinical practice of health professionals across Europe.

Disclosure of Interests: None declared

DOI: 10.1136/annrheumdis-2019-eular.8622

WEDNESDAY, 12 JUNE 2019

14:15:00 - 15:45:00

\section{Environmental influences on disease development}

\section{SP0011 HOW THE GUT INFLUENCES THE IMMUNE SYSTEM}

Mario Zaiss. Friedrich-Alexander University Erlangen-Nuremberg and Universitätsklinikum Erlangen, Department of Internal Medicine 3-Rheumatology and Immunology, Erlangen, Germany

Background: A disturbed gut microbiota composition has been associated with autoimmune diseases. Recent findings support the hypothesis that the onset of RA might be linked with the gut microbiota. Moreover, we could recently show that high fiber diets change the microbiota, increase SCFA levels and attenuate inflammatory arthritis and bone destruction ${ }^{(1)}$. However, direct mechanistic links between the gut microbiota and onset of autoimmune diseases remain largely unknown.

Objectives: Herein, we intended to identify if the intestinal barrier integrity could serve as a key checkpoint translating autoimmunity to inflammation.

Results: Restoration of the intestinal barrier in the pre-phase of arthritis using different approaches inhibited the development of arthritis.

Conclusion: In summary, our data identify a new preventive approach for the onset of autoimmune disease by specifically targeting impaired intestinal barrie function.

\section{REFERENCE:}

[1] Lucas S, Omata Y, Hofmann J, Böttcher M, Iljazovic A, Sarter K, Albrecht O, Schulz O, Krishnacoumar B, Krönke G, Herrmann M, Mougiakakos D, Strowig T, Schett G and Zaiss M M. (2018). Short-chain fatty acids regulate systemic bone mass and protect from pathological bone loss. Nat Commun 9: 55

Disclosure of Interests: None declared DOI: 10.1136/annrheumdis-2019-eular.8624 